\title{
Ehrlich-type Methods with King's Correction for the Simultaneous Approximation of Polynomial Complex Zeros
}

\author{
Roselaine Neves Machado ${ }^{1}$, Luiz Guerreiro Lopes ${ }^{2, *}$ \\ ${ }^{1}$ Federal Institute of Rio Grande do Sul, Bento Gonçalves Campus, 95700-206 Bento Gonçalves, RS, Brazil \\ ${ }^{2}$ Faculty of Exact Sciences and Engineering, University of Madeira, Penteada Campus, 9020-105 Funchal, Madeira Is., Portugal
}

Received August 7, 2019; Revised August 30, 2019; Accepted September 7, 2019

Copyright (C)2019 by authors, all rights reserved. Authors agree that this article remains permanently

open access under the terms of the Creative Commons Attribution License 4.0 International License

\begin{abstract}
There are many simultaneous iterative methods for approximating complex polynomial zeros, from more traditional numerical algorithms, such as the well-known third order Ehrlich-Aberth method, to the more recent ones. In this paper, we present a new family of combined iterative methods for the simultaneous determination of simple complex zeros of a polynomial, which uses the Ehrlich iteration and a correction based on King's family of iterative methods for nonlinear equations. The use of King's correction allows increasing the convergence order of the basic method from three to six. Some numerical examples are given to illustrate the convergence behaviour and effectiveness of the proposed sixth order Ehrlich-like family of combined iterative methods for the simultaneous approximation of simple complex polynomial zeros.
\end{abstract}

Keywords Polynomial Zeros, Simultaneous Iterative Methods, Combined Methods, Ehrlich Method

\section{Introduction}

The importance of the problem of finding polynomial zeros in the different branches of Science and Engineering has led to the development of many different methods for their numerical determination. There is also a significant interest in the development of new and efficient numerical iterative methods for determining the zeros of real and complex polynomials.

These methods can approximate the zeros of a polynomial either in a sequential or simultaneous manner. However, the determination of all zeros of a given polynomial by a sequential method involves repeated deflations, which can lead to very inaccurate results due to the problem of accumulating rounding errors when using finite precision floating-point arithmetic.

In turn, the simultaneous methods for polynomial zeros have the advantage of being inherently parallel and avoid the process of deflation, although these iterative methods usually need good initial approximations for all the zeros in order to converge.

In this work, a new family of numerical methods for the simultaneous approximation of simple complex polynomial zeros is presented. The proposed simultaneous methods are constructed on the basis of the well-known third order EhrlichAberth iteration [1, 6], combined with an iterative correction from the optimal fourth order two-step King's method for solving nonlinear equations [10].

\section{A family of high order Ehrlich-type simultaneous methods with King's correction}

\subsection{The third order Ehrlich method}

Let $P$ be a monic polynomial of degree $n$, with (real or complex) simple zeros $\zeta_{1}, \ldots, \zeta_{n}$,

$$
P(z)=z^{n}+a_{n-1} z^{n-1}+\cdots+a_{1} z+a_{0}=\prod_{j=1}^{n}\left(z-\zeta_{j}\right), a_{i} \in \mathbb{C} .
$$

Considering the correction term $N(z)=P(z) / P^{\prime}(z)$ from the second order Newton's method $\hat{z}=z-N(z)$ (where, for notation simplicity, $\hat{z}$ represents a new approximation for a zero $\zeta$ ) and taking its logarithmic derivative with respect to $z$, we obtain

$$
N(z)=\frac{P(z)}{P^{\prime}(z)}=\left[\frac{d}{d z} \log P(z)\right]^{-1}=\left(\sum_{j=1}^{n} \frac{1}{z-\zeta_{j}}\right)^{-1} .
$$


But we have

$$
\left(\sum_{j=1}^{n} \frac{1}{z-\zeta_{j}}\right)^{-1}=\left(\frac{1}{z-\zeta_{i}}+\sum_{\substack{j=1 \\ j \neq i}}^{n} \frac{1}{z-\zeta_{j}}\right)^{-1},
$$

and then

$$
N(z)=\frac{1}{\frac{1}{z-\zeta_{i}}+\sum_{\substack{j=1 \\ j \neq i}}^{n} \frac{1}{z-\zeta_{j}}} .
$$

From (4), we can easily derive the following fixed-point relation:

$$
\zeta_{i}=z-\frac{1}{\frac{1}{N(z)}-\sum_{\substack{j=1 \\ j \neq i}}^{n} \frac{1}{z-\zeta_{j}}} \quad(i=1, \ldots, n) .
$$

Now, let $z_{1}, \ldots, z_{n}$ be distinct approximations to the zeros $\zeta_{1}, \ldots, \zeta_{n}$ of the polynomial $P$.

Putting $\zeta_{i} \simeq \hat{z}_{i}$ in (5), where $\hat{z}_{i}$ is a new approximation for the zero $\zeta_{i}$, setting $z=z_{i}$, and substituting the zeros $\zeta_{j}$ by their approximations $z_{j}(j \neq i)$ on the right-hand side of the above identity, we obtain the well-known Ehrlich method (also known as Ehrlich-Aberth method or Maehly-Ehrlich-Aberth method) for the simultaneous approximation of simple polynomial zeros.

Therefore, Ehrlich's method for the simultaneous determination of polynomial zeros is given by

$$
\hat{z}_{i}=z_{i}-\frac{1}{\frac{1}{N\left(z_{i}\right)}-\sum_{\substack{j=1 \\ j \neq i}}^{n} \frac{1}{z_{i}-z_{j}}} \quad(i=1, \ldots, n) .
$$

This important result was independently derived by several different authors, including Maehly [11], Börsch-Supan [3], Dochev and Byrnev [5], Ehrlich [6], Weißenhorn [15], Aberth [1], and Farmer and Loizou [7]. $[2,6]$.

The Ehrlich method is cubically convergent for simple roots

\subsection{Ehrlich-type simultaneous methods with King's cor- rection}

It is evident from the fixed-point relation (5) that better approximations $z_{j}$ will produce more accurate approximations $\hat{z}_{i}$ for the zeros $\zeta_{i}$.

In order to achieve this, and aiming to improve the convergence rate and efficiency of the iterative scheme due to Ehrlich, we propose a family of simultaneous iterative methods constructed on the basis of the third order Ehrlich iteration, combined with a correction based on the optimal fourth order twostep King's method for nonlinear equations [10].

The well-known King's family of fourth order multipoint iterative methods for determining a simple root of a nonlinear equation $f(x)=0$ requires only two evaluations of the function and one of its first derivative per full iteration, and is defined by

$$
\begin{gathered}
y_{n}=x_{n}-\frac{f\left(x_{n}\right)}{f^{\prime}\left(x_{n}\right)}, \\
x_{n+1}=y_{n}-\frac{f\left(y_{n}\right)}{f^{\prime}\left(x_{n}\right)}\left[\frac{f\left(x_{n}\right)+\beta f\left(y_{n}\right)}{f\left(x_{n}\right)+(\beta-2) f\left(y_{n}\right)}\right],
\end{gathered}
$$

where $\beta \in \mathbb{C}$ is a parameter.

The family of simultaneous iterative methods here proposed is obtained by combining the scheme of the third order Ehrlich iteration (6) with an iterative correction term obtained from King's fourth order iteration (7), given by

$$
C_{K}\left(z_{j}\right)=\frac{P\left(y\left(z_{j}\right)\right)}{P^{\prime}\left(z_{j}\right)} \frac{P\left(z_{j}\right)+\beta P\left(y\left(z_{j}\right)\right)}{P\left(z_{j}\right)+(\beta-2) P\left(y\left(z_{j}\right)\right)},
$$

where

$$
y\left(z_{j}\right)=z_{j}-\frac{P\left(z_{j}\right)}{P^{\prime}\left(z_{j}\right)},
$$

and $\beta$ is a parameter.

Using the King approximation $z_{j}-C_{K}\left(z_{j}\right)$ in (6) instead of $z_{j}$, we obtain a new one-parameter family of Ehrlich-type simultaneous iterative methods with King's correction, defined by

$$
\hat{z}_{i}=z_{i}-\frac{1}{\frac{1}{N\left(z_{i}\right)}-\sum_{\substack{j=1 \\ j \neq i}}^{n} \frac{1}{z_{i}-z_{j}+C_{K}\left(z_{j}\right)}} \quad(i=1, \ldots, n),
$$

where $C_{K}\left(z_{j}\right)$ is the iterative correction appearing in (8).

\section{Order of convergence of the new fam- ily of combined methods}

In this section, we analyze the order of convergence of the proposed family of Ehrlich-type simultaneous iterative methods with King's correction, which is established in the following theorem.

Theorem 1. Let $z_{1}^{(0)}, \ldots, z_{n}^{(0)}$ be sufficiently close initial approximations to the simple zeros $\zeta_{1}, \ldots, \zeta_{n}$ of the polynomial $P$. Then, the order of convergence of the one-parameter family of iterative methods defined in (9) is six.

Proof. Consider the abbreviations

$$
\begin{gathered}
\lambda_{i, j}=z_{i}-z_{j}+C_{K}\left(z_{j}\right), \\
\theta_{i}=\sum_{\substack{j=1 \\
j \neq i}}^{n} \frac{z_{j}-\zeta_{j}-C_{K}\left(z_{j}\right)}{\left(z_{i}-\zeta_{j}\right) \lambda_{i, j}},
\end{gathered}
$$

and let $\epsilon_{i}$ and $\hat{\epsilon}_{i}$ be the errors of the numerical approximations defined as follows:

$$
\begin{aligned}
\epsilon_{i} & =z_{i}-\zeta_{i}, \\
\hat{\epsilon}_{i} & =\hat{z}_{i}-\zeta_{i} .
\end{aligned}
$$


Rearranging (9), we have

$$
\hat{z}_{i}=z_{i}-\frac{N\left(z_{i}\right)}{1-N\left(z_{i}\right) \sum_{\substack{j=1 \\ j \neq i}}^{n} \frac{1}{z_{i}-z_{j}+C_{K}\left(z_{j}\right)}}(i=1, \ldots, n) .
$$

Substituting (2) into (14), we obtain

$$
\hat{z}_{i}=z_{i}-\frac{\left(\sum_{j=1}^{n} \frac{1}{z_{i}-\zeta_{j}}\right)^{-1}}{1-\left(\sum_{j=1}^{n} \frac{1}{z_{i}-\zeta_{j}}\right)^{-1} \sum_{\substack{j=1 \\ j \neq i}}^{n} \frac{1}{z_{i}-z_{j}+C_{K}\left(z_{j}\right)}},
$$

and expanding this equation and simplifying, we get

$$
\hat{z}_{i}=z_{i}-\frac{1}{\sum_{j=1}^{n} \frac{1}{\left(z_{i}-\zeta_{j}\right)}-\sum_{\substack{j=1 \\ j \neq i}}^{n} \frac{1}{z_{i}-z_{j}+C_{K}\left(z_{j}\right)}} .
$$

However, when $i \neq j$, we can write

$$
\sum_{j=1}^{n} \frac{1}{\left(z_{i}-\zeta_{j}\right)}=\frac{1}{\left(z_{i}-\zeta_{i}\right)}+\sum_{\substack{j=1 \\ j \neq i}}^{n} \frac{1}{\left(z_{i}-\zeta_{j}\right)}
$$

Substituting (17) into (16) yields

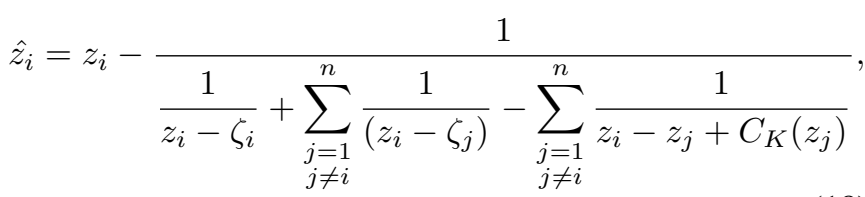

and substituting (12) into this equation gives

$$
\hat{z_{i}}=z_{i}-\frac{1}{\frac{1}{\epsilon_{i}}+\sum_{\substack{j=1 \\ j \neq i}}^{n} \frac{1}{\left(z_{i}-\zeta_{j}\right)}-\sum_{\substack{j=1 \\ j \neq i}}^{n} \frac{1}{z_{i}-z_{j}+C_{K}\left(z_{j}\right)}} .
$$

But, by (12) and (13), we have

$$
\hat{\epsilon}_{i}+\zeta_{i}=\epsilon_{i}+\zeta_{i}-\frac{1}{\frac{1}{\epsilon_{i}}+\sum_{\substack{j=1 \\ j \neq i}}^{n} \frac{1}{z_{i}-\zeta_{j}}-\sum_{\substack{j=1 \\ j \neq i}}^{n} \frac{1}{z_{i}-z_{j}+C_{K}\left(z_{j}\right)}} .
$$

Simplifying and replacing the denominator of the second summation by (10), we get

$$
\hat{\epsilon_{i}}=\epsilon_{i}-\frac{1}{\frac{1}{\epsilon_{i}}+\sum_{\substack{j=1 \\ j \neq i}}^{n} \frac{1}{z_{i}-\zeta_{j}}-\sum_{\substack{j=1 \\ j \neq i}}^{n} \frac{1}{\lambda_{i, j}}} .
$$

Multiplying and dividing the last equation by $\epsilon_{i}$, we have

$$
\hat{\epsilon_{i}}=\epsilon_{i}-\frac{\epsilon_{i}}{1+\epsilon_{i}\left(\sum_{\substack{j=1 \\ j \neq i}}^{n} \frac{1}{z_{i}-\zeta_{j}}-\sum_{\substack{j=1 \\ j \neq i}}^{n} \frac{1}{\lambda_{i, j}}\right)},
$$

and joining the two summations together, we obtain

$$
\hat{\epsilon_{i}}=\epsilon_{i}-\frac{\epsilon_{i}}{1+\epsilon_{i}\left(\sum_{\substack{j=1 \\ j \neq i}}^{n} \frac{\lambda_{i, j}-\left(z_{i}-\zeta_{j}\right)}{\left(z_{i}-\zeta_{j}\right) \lambda_{i, j}}\right)} .
$$

Considering (10) again, we have

$$
\hat{\epsilon_{i}}=\epsilon_{i}-\frac{\epsilon_{i}}{1+\epsilon_{i}\left(\sum_{\substack{j=1 \\ j \neq i}}^{n} \frac{z_{i}-z_{j}+C_{K}\left(z_{j}\right)-z_{i}+\zeta_{j}}{\left(z_{i}-\zeta_{j}\right) \lambda_{i, j}}\right)},
$$

and simplifying, we obtain

$$
\hat{\epsilon_{i}}=\epsilon_{i}-\frac{\epsilon_{i}}{1+\epsilon_{i}\left(\sum_{\substack{j=1 \\ j \neq i}}^{n} \frac{C_{K}\left(z_{j}\right)-z_{j}+\zeta_{j}}{\left(z_{i}-\zeta_{j}\right) \lambda_{i, j}}\right)},
$$

where the summation that appears within parentheses in this equation is, according to (11), equal to $-\theta_{i}$.

By making the proper substitution in (25), we get

$$
\hat{\epsilon_{i}}=\epsilon_{i}-\frac{\epsilon_{i}}{1+\epsilon_{i}\left(-\theta_{i}\right)} .
$$

Reducing to the common denominator and simplifying, we finally obtain

$$
\hat{\epsilon_{i}}=\frac{-\epsilon_{i}^{2} \theta_{i}}{1-\epsilon_{i} \theta_{i}} .
$$

By the assumption of the theorem, the errors are small enough in modulus, and thus it can be assumed that $\epsilon_{i}=$ $\mathcal{O}_{m}\left(\epsilon_{j}\right)$ and $\hat{\epsilon}_{i}=\mathcal{O}_{m}\left(\hat{\epsilon}_{j}\right)$ for $i, j \in\{1, \ldots, n\}$, where this notation means that the moduli of the pairs of complex numbers involved have the same order, that is, $\left|\epsilon_{i}\right|=\mathcal{O}\left(\left|\epsilon_{j}\right|\right)$ and $\left|\hat{\epsilon}_{i}\right|=\mathcal{O}\left(\left|\hat{\epsilon}_{j}\right|\right)$, where the symbol $\mathcal{O}$ indicates the order of magnitude.

Analyzing (10) and (11), we observe that, for sufficiently close approximations to the corresponding zeros, the expression in the denominator of (11) is bounded and tends to $\left(\zeta_{i}-\zeta_{j}\right)^{2}$, for $i \neq j$.

Considering this, and taking into account that each method of the family of King's methods is of fourth order of convergence [10], i.e., for these methods, $\hat{z}-\zeta=\mathcal{O}_{m}\left((z-\zeta)^{4}\right)$, we find that

$$
\theta_{i}=\mathcal{O}_{m}\left(\epsilon^{4}\right) .
$$

Using this result with (27), we conclude that

$$
\hat{\epsilon}=\mathcal{O}_{m}\left(\epsilon^{6}\right) .
$$


Thus, the order of convergence of the family of Ehrlich-type simultaneous iterative methods with King's correction (9) is six.

\section{Numerical examples}

In this section, some numerical examples are presented to illustrate the convergence behaviour and effectiveness of the proposed family of simultaneous iterative methods.

It is important to note here that not all values of the parameter $\beta$ lead to convergence of the fourth order King's method (7) [4]. Some convenient values of this parameter can be found in the literature, including $\beta=-1 / 2[10], \beta=-9 / 2$, $\beta=3.9+0.1 i[4]$ and $\beta=3-2 \sqrt{2} i[12]$.

In the three examples here considered, we have adopted the value $\beta=-7 / 10$ for this parameter, which was determined through a computational parameter optimization.

In the examples presented, the initial approximations were chosen by using Aberth's approach [1]. The starting approximations to the zeros of each polynomial calculated by the Aberth's initialization procedure are equidistantly distributed along the circumference $\left|z+a_{1} / n\right|=r_{0}$, where $a_{1}$ is the coefficient of the second leading term of the monic polynomial considered, $n$ is the polynomial degree, and $r_{0}$ is the radius of a disk centered at the origin of the complex plane containing all the zeros of the polynomial, obtained as usual (see, e.g., [13]) by applying the Guggenheimer's upper bound for the zeros [9].

The numerical tolerance was set to $1 \times 10^{-12}$ and the maximum number of iterations was fixed to 50 .

Example 1. The first example considered is that of a complex polynominal of degree 10 , extracted from [8], with zeros \pm 1 , $\pm i, \pm \sqrt{2} / 2(1 \pm i), 2 i$, and $3 i$ :

$P_{1}(z)=z^{10}-5 i z^{9}-6 z^{8}-z^{2}+5 i z+6$.

Figure 1 presents the trajectories of approximations for the polynomial $P_{1}(z)$ generated by the simultaneous iterative method (9) with $a_{1}=-5 i, r_{0}=10$ and $\beta=-7 / 10$.

The requested accuracy of $10^{-12}$ was achieved in eigth iterations, while Ehrlich's method (6), on which the proposed method is based, required 14 iterations.

Example 2. In the second example, the proposed method (9), with $\beta=-7 / 10$, was applied to a real polynomial of degree 15 , extracted from [14], which has only a real zero:

$P_{2}(z)=z^{15}+z^{14}+1$.

The trajectories of approximations for the polynomial $P_{2}(z)$, generated by the method (9) with $a_{1}=1, r_{0}=2$ and $\beta=$ $-7 / 10$, are presented in Figure 2.

In this case, the proposed combined iterative method required five iterations to obtain approximations to the zeros of the given polynomial with the requested accuracy.

For comparison, Ehrlich's method needed nine iterations to achieve the same accuracy.

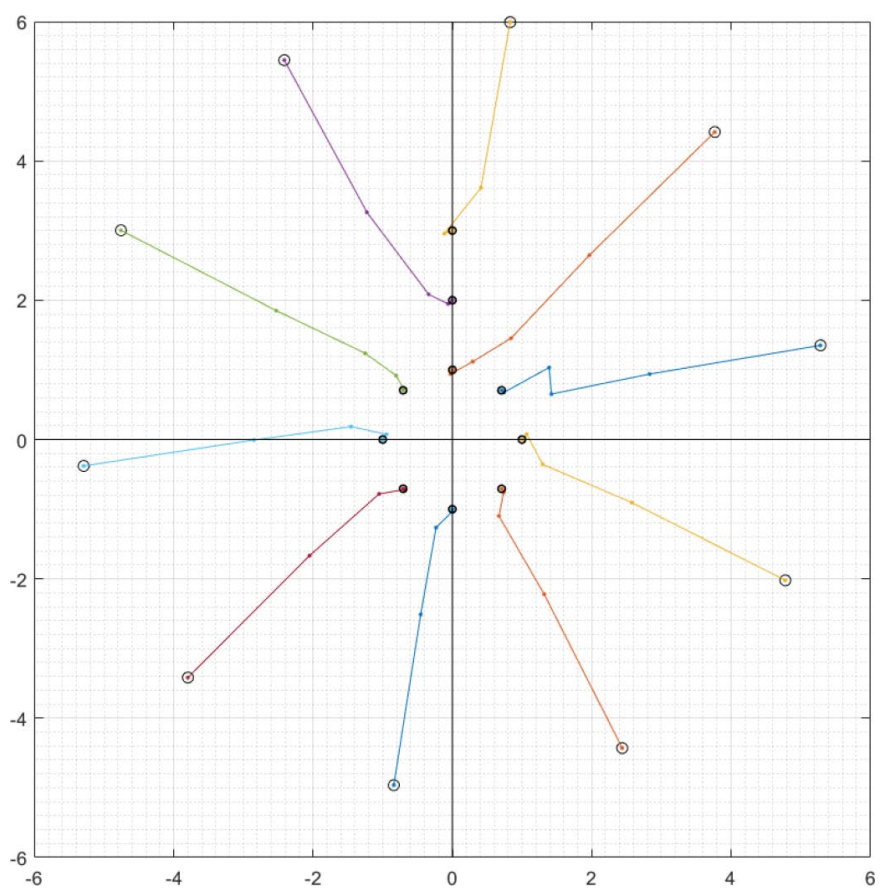

Figure 1. Trajectories of approximations for Example 1.

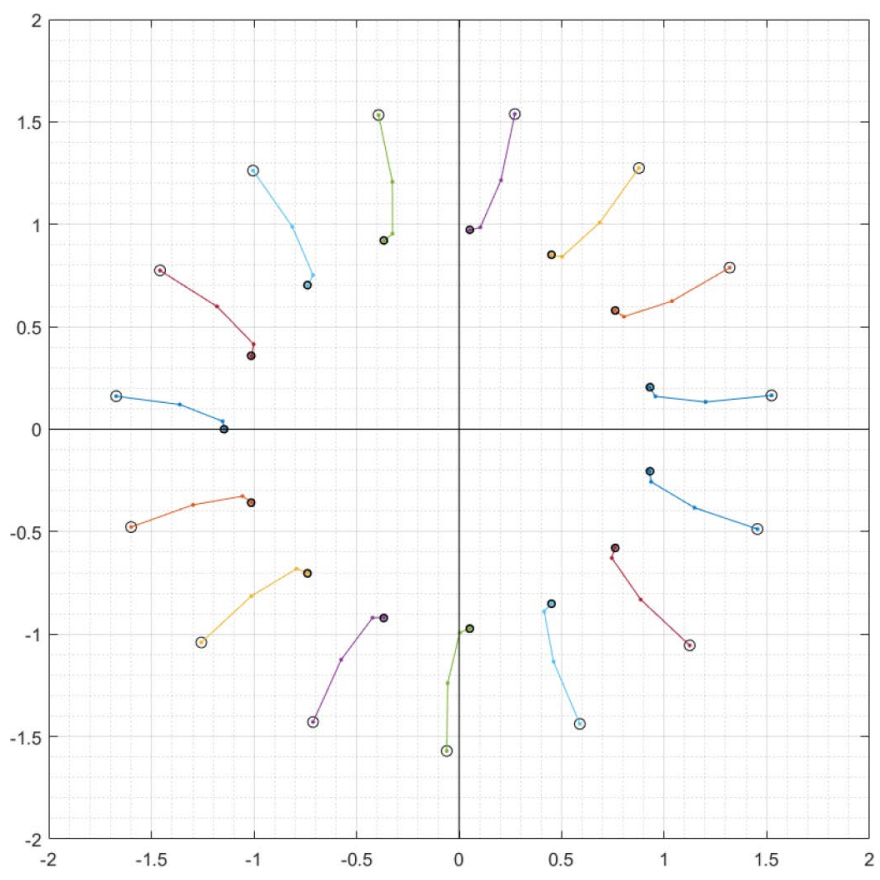

Figure 2. Trajectories of approximations for Example 2. 
Example 3. The last example here considered is a real polynomial of degree 20, extracted from [16], known as the scaled Wilkinson polynomial:

$P_{3}(z)=\prod_{k=1}^{20}\left(z-\frac{k}{20}\right)$.

Figure 3 shows the trajectories of approximations for the scaled Wilkinson polynomial generated by the method (9) with $a_{1}=-10.5, r_{0}=21$ and $\beta=-7 / 10$.

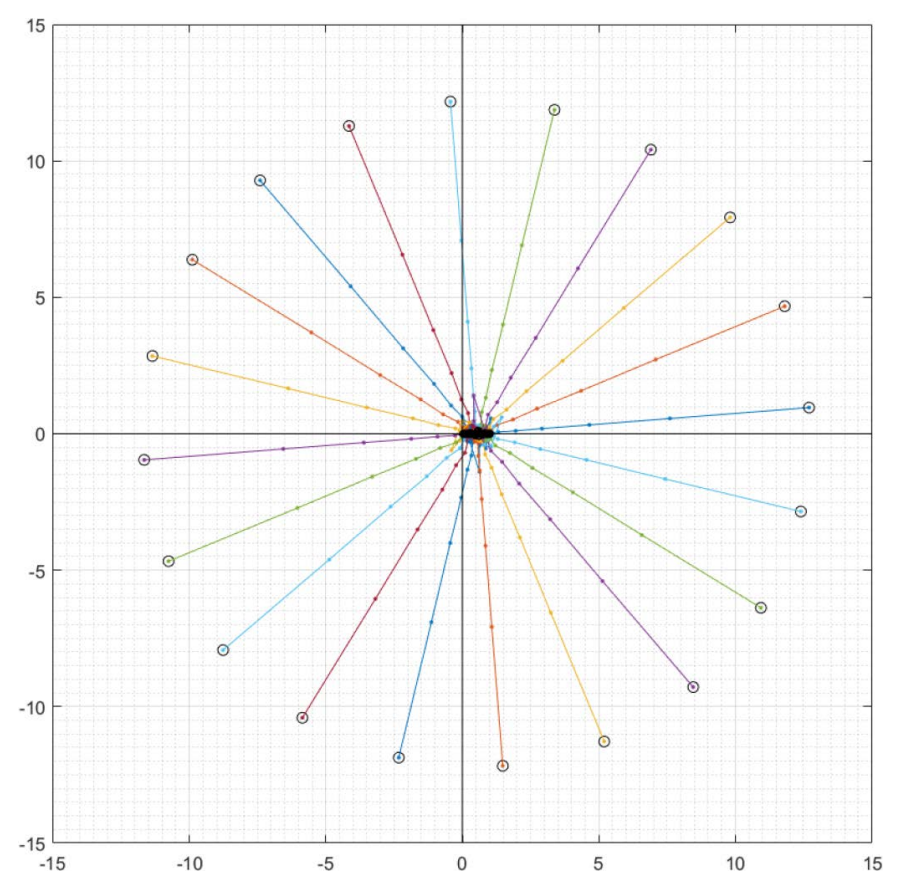

Figure 3. Trajectories of approximations for Example 3.

A more detailed view of the trajectories of approximations for the same polynomial corresponding to the last 11 iterations generated by the proposed simultaneous method is shown in Figure 4.

The third order Ehrlich method needed 45 iterations to reach the required accuracy, whereas the proposed method (9), with $\beta=-7 / 10$, performed only 15 iterations to reach the same desired accuracy.

Table 1 contains a summary of the results obtained, showing the number of iterations required by the simultaneous iterative methods (6) and (9) to produce approximations to the zeros of the polynomials of Examples 1 to 3 with the requested accuracy of $10^{-12}$.

Table 1. Number of iterations necessary to obtain approximations accurate to within $10^{-12}$

\begin{tabular}{|l|c|c|c|c|}
\hline Method & \multirow{2}{*}{ Eq. } & \multicolumn{3}{|c|}{ No. of iterations } \\
\cline { 3 - 5 } & & $P_{1}$ & $P_{2}$ & $P_{3}$ \\
\hline \hline Ehrlich & $(6)$ & 14 & 9 & 45 \\
Ehrlich-King $(\beta=-7 / 10)$ & $(9)$ & 8 & 5 & 15 \\
\hline
\end{tabular}

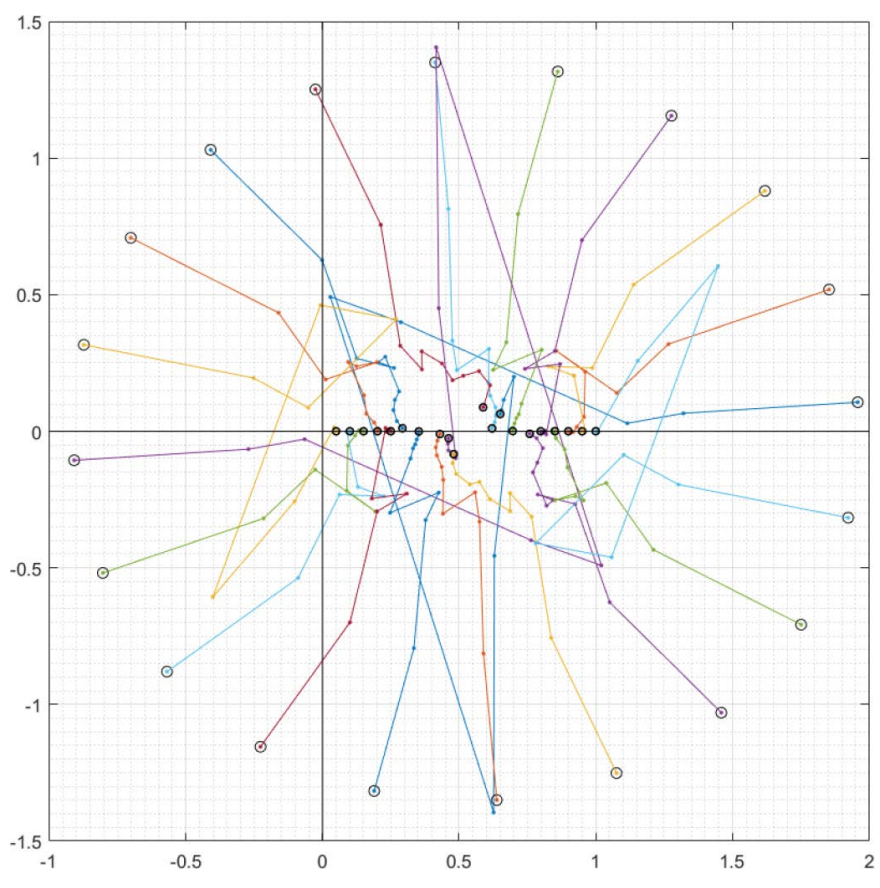

Figure 4. Detail of the trajectories of approximations for Example 3.

\section{Conclusion}

In this paper, a new sixth order family of combined iterative methods for the simultaneous approximation of simple complex zeros of a polynomial was introduced and discussed.

The proposed one-parameter family of simultaneous methods was constructed on the basis of the third order Ehrlich iteration, combined with an iterative correction based on King's family of optimal fourth order iterative methods for solving nonlinear equations.

It was proved that the use of King's correction allows to increase the order of convergence of the basic simultaneous method from three to six.

The results of some numerical examples corroborate the theoretical analysis and illustrate the effectiveness and rapid convergence of the proposed Ehrlich-type methods with King's correction for the simultaneous approximation of polynomial complex zeros.

\section{Acknowledgement}

This work was carried out as part of the first author's $\mathrm{PhD}$ research at the University of Madeira, Portugal, and was supported by the Federal Institute of Rio Grande do Sul (IFRS), Brazil.

\section{REFERENCES}

[1] O. Aberth. Iteration methods for finding all zeros of a polynomial simultaneously, Mathematics of Computation, Vol. 27, No. 122, 339-344, 1973. doi:10.1090/S0025-57181973-0329236-7. 
[2] G. Alefeld, J. Herzberger. On the convergence speed of some algorithms for the simultaneous approximation of polynomial roots, SIAM Journal on Numerical Analysis, Vol. 11, No. 2, 237-243, 1974. doi:10.1137/0711023.

[3] W. Börsch-Supan. A posteriori error bounds for the zeros of polynomials, Numerische Mathematik, Vol. 5, No. 1, 380-398, 1963. doi:10.1007/BF01385904.

[4] A. Cordero, J. García-Maimó, J. R. Torregrosa, M. P. Vassileva, P. Vindel. Chaos in King's iterative family, Applied Mathematics Letters, Vol. 26, No. 8, 842-848, 2013. doi:10.1016/j.aml.2013.03.012.

[5] K. Dochev, P. Byrnev. Certain modifications of Newton's method for the approximate solution of algebraic equations, USSR Computational Mathematics and Mathematical Physics, Vol. 4, No. 5, 174-182, 1964. doi:10.1016/00415553(64)90148-X.

[6] L. W. Ehrlich. A modified Newton method for polynomials, Communications of the ACM, Vol. 10, No. 2, 107-108, 1967. doi:10.1145/363067.363115.

[7] M. R. Farmer, G. Loizou. A class of iteration functions for improving, simultaneously, approximations to the zeros of a polynomial, BIT, Vol. 15, No. 3, 250-258, 1975. doi:10.1007/BF01933657.

[8] I. Gargantini. Parallel Laguerre iterations: the complex case, Numerische Mathematik, Vol. 26, No. 3, 317-323, 1976. doi:10.1007/BF01395948.

[9] H. Guggenheimer. Bounds for roots of algebraic equations, Archiv der Mathematik, Vol. 31, No. 1, 568-569, 1978. doi:10.1007/BF01226493.
[10] R. F. King. A family of fourth order methods for nonlinear equations, SIAM Journal of Numerical Analysis, Vol. 10, No. 5, 876-879, 1973. doi:10.1137/0710072.

[11] H. J. Maehly. Zur iterativen Auflösung algebraischer Gleichungen, Zeitschrift für Angewandte Mathematik und Physik, Vol. 5, No. 3, 260-263, 1954. doi:10.1007/BF01600333.

[12] B. Neta, M. Scott, C. Chun. Basins of attraction for several methods to find simple roots of nonlinear equations, Applied Mathematics and Computation, Vol. 218, No. 21, 10548-10556, 2012. doi:10.1016/j.amc.2012.04.017.

[13] M. S. Petković, S. Ilić, I. Petković. A posteriori error bound methods for the inclusion of polynomial zeros, Journal of Computational and Applied Mathematics, Vol. 208, No. 2, 316-330, 2007. doi:10.1016/j.cam.2006.09.014.

[14] P. D. Proinov, M. T. Vasileva. On the convergence of high-order Ehrlich-type iterative methods for approximating all zeros of a polynomial simultaneously, Journal of Inequalities and Applications, Vol. 2015, Art. No. 336, 2015. doi:10.1186/s13660-0150855-5.

[15] F. Weißenhorn. Ein Beitrag zur Bestimmung der Nullstellen aus einer Polynom in Summenform und aus der Summe von Polynomen in Produktform, Archiv für Elektronik und Übertragungstechnik, Vol. 24, 372-378, 1970.

[16] H. Zhang. Numerical condition of polynomials in different forms, Electronic Transactions on Numerical Analysis, Vol. 12, 66-87, 2001. 